\title{
Idiopathic Superficial Thrombophlebitis and the Incidence of Cancer in Primary Care Patients
}

\author{
Frederiek F. van Doormaal, $M D^{1}$ \\ Selma Atalay ${ }^{1}$ \\ Henk J. Brouwer, $\mathrm{MsC}^{2}$ \\ Eit-Frits van der Velde, $M D^{2}$ \\ Harry R. Büller, $M D^{1}$ \\ Henk C. van Weert, $M D^{2}$ \\ ${ }^{1}$ Department of Vascular Medicine, \\ Academic Medical Center Amsterdam, \\ The Netherlands \\ ${ }^{2}$ Department of General Practice, \\ Academic Medical Center of Amsterdam, \\ The Netherlands
}

\begin{abstract}
PURPOSE The association of spontaneous venous thromboembolism with occult malignancy is well established. Less clear is the incidence of subsequent cancer in patients with superficial thrombophlebitis. We wanted to determine the incidence of cancer after an episode of spontaneous superficial thrombophlebitis in a large general practice population.
\end{abstract}

METHODS The objective of this study was to assess the incidence of newly diagnosed malignancies in patients within 2 years after the diagnosis of a spontaneous episode of superficial thrombophlebitis and to compare this incidence with nonexposed matched control patients and the Dutch population. The patients and their controls were identified by a search in the electronic patient records of 5 primary health care centers in Amsterdam, the Netherlands. A standardized morbidity ratio was calculated using data of the Dutch cancer registry.

RESULTS A total number of 277 patients with superficial thrombophlebitis were identified, of which 250 patients had no cancer at study entry. In 5 of these 250 patients (2\%; 95\% confidence interval [Cl], 1\%-5\%), a new malignancy was diagnosed within 2 years after their superficial thrombophlebitis compared with $2 \%(95 \% \mathrm{Cl}, 1 \%-4 \%)$ in the control group. The standardized morbidity ratio was $1.1(95 \% \mathrm{Cl}, 0.5-2.7)$. A recurrent episode of superficial thrombophlebitis was observed in 18 of the 250 patients, and in 1 patient cancer was diagnosed within 24 months after the first episode of superficial thrombophlebitis.

CONCLUSION We conclude that a single episode of unprovoked superficial thrombophlebitis diagnosed by a family physician is not associated with an increased risk of subsequent cancer.

Ann Fam Med 2010;8:47-50. doi:10.1370/afm.1058.

\section{INTRODUCTION}

T n 1865, Armand Trousseau described an association between recurrent superficial thrombophlebitis and occult malignancy, which is now termed the Trousseau syndrome. ${ }^{1}$ It is characterized by a recurrent and migratory pattern of inflammation of superficial veins, frequently in unusual sites such as the arm or chest. Since then, many studies reported on the association between venous thromboembolism and cancer. A recent study observed a mean $10 \%$ (95\% confidence interval [CI], 8.6\%-11.3\%) incidence of malignancy within the first 12 months after the diagnosis of an unprovoked deep vein thrombosis of the leg or pulmonary embolism. ${ }^{2}$ Venous thromboembolism is believed to be at least partially provoked by the still occult cancer. Although the incidence of newly diagnosed cancer is increased until years after the diagnosis of unprovoked venous thromboembolism, most cancers are diagnosed within the first months. ${ }^{3,4}$ Numerous textbooks state that not only deep vein thrombosis or pulmonary embolism but also superficial venous thrombophlebitis (SVTP) is associ- 
ated with occult malignancy. Careful analysis of the literature shows, however, that so far only case reports have described this association.

We therefore performed a study to determine the incidence of newly diagnosed malignancies during a 2-year follow-up period after a first episode of unprovoked SVTP in a large general practice population. The incidence of cancer was compared with that in a cohort of practice-, age-, and sex-matched control patients without SVTP and to the incidence of cancer in the Dutch population.

\section{METHODS}

In the Dutch health care system, every citizen is enrolled by a family physician, who keeps health records and functions as a gatekeeper to secondary care. This family physician is consulted for any health problem for which medical care is needed. Family physicians treat approximately $94 \%$ of the health problems themselves and refer, if necessary. ${ }^{5}$

The electronic patient records of 5 health care centers (28 family physicians) are stored anonymously at the Department of General Practice in the Academic Medical Center, Amsterdam, the Netherlands, and were available for the study analyses. In this register is recorded relevant information of all contacts between the physician and patients. The International Classification of Primary Care (ICPC) is used for diagnostic coding, the World Health Organization's Anatomical Therapeutic Chemical Classification System codes are used for medication coding. We analyzed anonymous data of approximately 38,000 adult patients for the study reported here The inception period was from January 1995 until December 2004.

\section{Identification of Case and Control Groups}

The medical records were searched for episodes of SVTP using diagnostic coding and truncated keywords $\left(\mathrm{t}(\mathrm{h})\right.$ romb* $^{*}$, phleb* and fleb*). Family physicians diagnosed SVTP clinically according to the rules of ICPC: "signs of inflammation along a superficial vein." The medical records were checked by 2 investigators independently to determine whether the record did report SVTP. Where there was more than 1 possible diagnosis, SVTP had to be first on the list to be included in the study. The date of diagnosis of SVTP was taken as the index date. SVTP associated with venous catheters or recent surgery as noted in the medical records were considered to be provoked and therefore excluded. We manually reviewed all consultations during the month after the event to exclude misclassified cases. For each SVTP patient, we selected 2 control patients for whom there was no history of venous thrombosis or SVTP, matched for family physician, sex, and age. No other patient characteristics to control for possible cofounding could be extracted from the source database.

\section{Follow-up}

Family physicians register all malignancies with the date of diagnosis in the problem lists of the medical records of all patients. Because of the seriousness of the disease, it was assumed that all diagnosed malignancies were captured in this database. Additionally, the medical records of both the SVTP group and the control group were searched manually for any listing of cancer. The patients in whom cancer was diagnosed before SVTP was diagnosed were categorized separately. Study patients and control patients were observed for 24 months.

The cumulative incidence of cancer per sex and age category of the SVTP population was also compared with the incidence of cancer per sex and age of the overall Dutch population using data of the national cancer registry. This registration collects data from cancer patients; it includes tumor type, date of diagnosis, and tumor stage and is linked to the international agency for research on cancer. ${ }^{6}$ A standardized morbidity ratio (SMR) was calculated using the Stata 8 software package (StataCorp LP, College Station, Texas).

\section{RESULTS}

Among a total of 880 cases reviewed for SVTP, provoked SVTP was recorded for 27 patients. The total number of patients with a spontaneous episode of SVTP was 277 , of whom $26 \%$ were men, and their mean age was 59 years. The matched control group consisted of 553 patients, of whom $26 \%$ were also men, and their mean age was 58 years. For 1 female patient born in 1907, we were able to find only 1 control patient of the same age and sex with the same family physician. Of all patients with unprovoked SVTP, cancer was diagnosed in 27 (10\%; 95\% CI, 7\%-14\%) before the episode of SVTP, whereas in 49 of the control patients $(9 \% ; 95 \% \mathrm{CI}, 7 \%-12 \%)$, a malignant process was diagnosed before the index date.

We retrieved complete follow-up data for 24 months after the index date in $243(88 \%)$ patients with SVTP and 469 (85\%) control patients. The other patients were either lost to follow-up $(n=83)$ or died within 24 months of the index date $(n=35)$, with similar rates for both groups. For the patients who did not complete 24-month follow-up consultations, the mean follow-up period of the SVTP patients was 12 months compared with a mean follow-up of 11 months in the control patients. 


\begin{tabular}{|c|c|c|c|c|c|}
\hline \multirow[b]{2}{*}{ Follow-up and Outcome } & \multicolumn{2}{|c|}{ SVTP Patients } & \multicolumn{2}{|c|}{ Control Group } & \multirow{2}{*}{$\begin{array}{l}\text { Absolute } \\
\text { Difference } \\
\%(95 \% \mathrm{Cl})\end{array}$} \\
\hline & No. $(\%)$ & $95 \% \mathrm{Cl}$ & No. (\%) & $95 \% \mathrm{Cl}$ & \\
\hline $\begin{array}{l}\text { Patients with incomplete } \\
\text { follow-up }\end{array}$ & $34(12)$ & $8-16$ & $84(15)$ & $12-18$ & -3 (-8 to 2$)$ \\
\hline $\begin{array}{l}\text { Deaths within } 24 \text { months after } \\
\text { index date }\end{array}$ & $16(6)$ & $3-9$ & $19(3)$ & $2-5$ & $2(-1$ to 5$)$ \\
\hline $\begin{array}{l}\text { Patients with newly diagnosed } \\
\text { cancer within } 24 \text { months } \\
\text { after index date }\end{array}$ & $5(2)$ & $1-5$ & $10(2)$ & $1-4$ & $0(-2$ to 2$)$ \\
\hline $\begin{array}{l}\text { Recurrent SVTP in patients with- } \\
\text { out cancer at study entry }\end{array}$ & $18(7)$ & $4-11$ & NA & NA & NA \\
\hline
\end{tabular}

In 5 of 250 patients with SVTP who did not have cancer at study entry $(2 \% ; 95 \% \mathrm{CI}, 1 \%-5 \%)$, a new malignancy was diagnosed within 24 months after the diagnosis of spontaneous SVTP compared with 10 new malignancies in the 504 control patients who did not have cancer at study entry $(2 \% ; 95 \% \mathrm{CI}, 1 \%-4 \%)$. The outcomes are summarized in Table 1. Two of the 5 patients with newly diagnosed cancer in the SVTP group died within the 24 -month follow-up period compared with 2 of the 10 control patients with a newly diagnosed malignancy. None of the other cancer patients in the SVTP and control groups were lost to follow-up. The types of cancer are described in Table 2. Compared with the overall Dutch population, no difference was observed in the incidence of cancer in 2 years after the index date, SMR $=1.1(95 \% \mathrm{CI}$, $0.5-2.7)$.

Of all 245 patients with SVTP who did not have cancer at study entry or during follow-up, 5 patients (2\%) died before the end of the 24-month follow-up period (Table 1). In the control group 11 (2\%) of the 494 patients died within 24 months after the index date. No death was associated with cancer.

Of the 250 patients with SVTP who did not have cancer at study entry, 18 patients $(7 \%$; 95\% CI, 4\%-

Table 2. Malignancies Diagnosed Within 2 Years After the Index Date

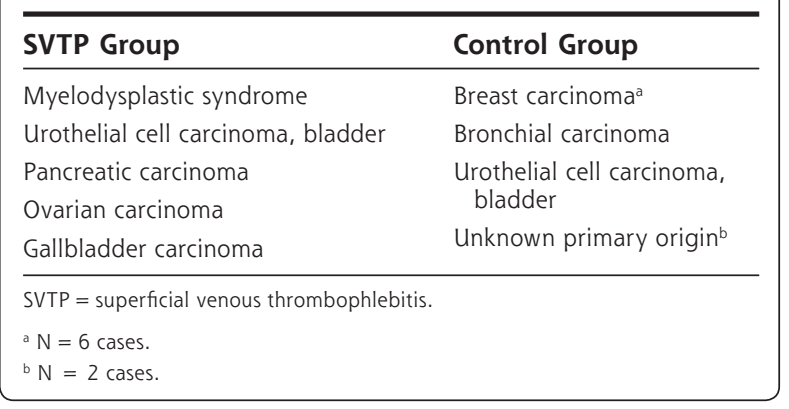

\section{DISCUSSION}

$11 \%)$ experienced at least 1 recurrent episode of SVTP during the 2-year follow-up period. The mean age of these patients was 62 years (35-87 years) and 14 of them were women. The median period between the first episode of SVTP and its recurrence was 12 months (0.6-23.0 months). A malignancy was diagnosed during the follow-up period in 1 patient with recurrent SVTP (6\%; 95\% CI, 0.1\%-27\%).

Careful review of his medical records (including hospital records) showed that a kidney cell carcinoma was present (but not diagnosed) when he had his first episode of SVTP diagnosed.

In this case-control study we found no increased incidence of cancer after the diagnosis of a first episode of unprovoked SVTP. During the 2-year observation period after a diagnosis of SVTP, the cumulative incidence of cancer was $2 \%(95 \% \mathrm{CI}, 1 \%-5 \%)$. These findings were confirmed after comparing the incidence of new cancers in the patients who had SVTP with the incidence of new cancers in the overall Dutch population (SMR 1.1; 95\% CI, 0.5\%-2.7\%). Two or more episodes of SVTP might be associated with a higher risk of cancer, but in the present cohort it occurred in only 1 patient.

We acknowledge some strengths and limitations of this work. To our knowledge this empirical study is the first in general practice that determines the possible association of spontaneous SVTP with cancer. A sensitive search strategy was used within the registry to identify patients with SVTP, which we believe made the identification of patients with SVTP almost complete. Furthermore, we believe that the recording system made complete ascertainment of diagnosed cancers likely, especially given the importance of this disease for daily practice. Finally, we used the same search strategy for both groups and matched for family physician to ensure that cases and controls were comparable.

A limitation of the study is the lack of objective assessment of the diagnosis of SVTP. Family physicians diagnose SVTP clinically. An objective assessment of the diagnosis is performed only when deep venous thrombosis is also suspected. To diminish the number of misclassifications, we checked the medical records of these patients for 4 weeks after the date of diagnosis 
of SVTP with the assumption that family physicians would change their diagnosis when it proved wrong. Another limitation of this study is the relatively high rate of patients lost to follow-up: of $6 \%$ in the SVTP group and $12 \%$ in the control group. The diagnosis of new malignancies in these patients cannot be excluded. Even so, considering the higher number of patients lost to follow-up in the control group, it is more likely to bias toward the no-difference observation. Furthermore, the sample size was insufficient to detect a small difference between case and control groups. For example, 1,245 patients with SVTP would be needed to detect a difference of $2 \%$, assuming a $4 \%$ incidence of cancer in the SVTP group compared with a $2 \%$ incidence of cancer in the control group $(1$-sided $\alpha=$ $0.05, \beta=0.1)$. Such a small difference, however, would probably not be of great clinical relevance. Finally, more information on patient characteristics could not be extracted from the source database, which impaired analyses on potential confounders.

We conclude that a first episode of unprovoked SVTP diagnosed by a family physician is not associated with an increased risk of cancer within the next 2 years relative to unaffected patients or the general public. As a result, it is unlikely that patients with SVTP will have an occult malignancy at the time of their diagnosis. Recurrent SVTP was uncommon in this sample, allowing no conclusion about whether recurrent SVTP is associated with cancer, as case reports have suggested.

To read or post commentaries in response to this article, see it online at http://www.annfammed.org/cgi/content/full/8/1/47.
Submitted March 4, 2009; submitted, revised, May 20, 2009; accepted June 10, 2009.

Keywords: superficial thrombophlebitis; cancer; primary care

The main results of this study have been presented at the International Conference on Thrombosis and Hemostasis Issues in Cancer, Begamo, Italy, October 2007.

Acknowledgments: We thank Jeanette Blom from the Department of Clinical Epidemiology, Leiden University Medical Center, The Netherlands for her help with Stata, and Geerte van Sluis from the Department of Vascular Medicine, Academic Medical Center Amsterdam, The Netherlands, for her assistance. We also thank the general practitioners involved in the Network of General Practitioners of the Academic Medical Centre/University of Amsterdam (HAG-net-AMC) for their continuous efforts to keep the electronic medical records updated.

\section{References}

1. Varki A. Trousseau's syndrome: multiple definitions and multiple mechanisms. Blood. 2007;110(6):1723-1729.

2. Carrier M, Le Gal G, Wells PS, Fergusson D, Ramsay T, Rodger MA. Systematic review: the Trousseau syndrome revisited: should we screen extensively for cancer in patients with venous thromboembolism? Ann Intern Med. 2008;149(5):323-333.

3. Blom JW, Doggen CJ, Osanto S, Rosendaal FR. Malignancies, prothrombotic mutations, and the risk of venous thrombosis. JAMA. 2005;293(6):715-722.

4. Sørensen HT, Mellemkjaer L, Steffensen FH, Olsen JH, Nielsen GL. The risk of a diagnosis of cancer after primary deep venous thrombosis or pulmonary embolism. N Engl J Med. 1998;338(17):1169-1173.

5. van Weert H, Dolan G, Wichers I, de Vries C, ter Riet G, Buller H; van WH. Spontaneous superficial venous thrombophlebitis: does it increase risk for thromboembolism? A historic follow-up study in primary care. J Fam Pract. 2006;55(1):52-57.

6. van Dam FS. [The hidden treasure-chests of the regional cancer registries] [article in Dutch]. Ned Tijdschr Geneeskd. 2004;148(17):814-815. 